University of Nebraska - Lincoln

DigitalCommons@University of Nebraska - Lincoln

$12-15-1990$

\title{
The relationship of the virtual orbitals of self-consistent-field theory to temporary negative ions in electron scattering from molecules
}

Donghai Chen

University of Nebraska-Lincoln

Gordon A. Gallup

University of Nebraska-Lincoln, ggallup1@unl.edu

Follow this and additional works at: https://digitalcommons.unl.edu/physicsgallup

Part of the Physics Commons

Chen, Donghai and Gallup, Gordon A., "The relationship of the virtual orbitals of self-consistent-field theory to temporary negative ions in electron scattering from molecules" (1990). Gordon Gallup Publications. 5. https://digitalcommons.unl.edu/physicsgallup/5

This Article is brought to you for free and open access by the Research Papers in Physics and Astronomy at DigitalCommons@University of Nebraska - Lincoln. It has been accepted for inclusion in Gordon Gallup Publications by an authorized administrator of DigitalCommons@University of Nebraska - Lincoln. 


\title{
The relationship of the virtual orbitals of self-consistent-field theory to temporary negative ions in electron scattering from molecules
}

\author{
Donghai Chena) and G. A. Gallup \\ Department of Chemistry, University of Nebraska, Lincoln, Nebraska 68588-0304
}

(Received 25 June 1990; accepted 10 September 1990)

\begin{abstract}
The theory given here shows how certain of the virtual orbitals of self-consistent field (SCF) theory provide a natural ingredient for constructing basis functions to study the shape resonances associated with temporary molecular ions. We give specific calculations of resonant behavior in $\mathrm{H}_{2}, \mathrm{~N}_{2}$, ethylene, cyclopropene, and cyclobutene. In addition, we show how the theory can rationalize the relationship between Koopmans' theorem values of the energy of a resonance and the experimental values.
\end{abstract}

\section{INTRODUCTION}

Frontier orbital theory (FOT) has come to provide important insights for analyzing the interactions between molecules, in general, and how they react when the energy is great enough. ${ }^{1}$ Both theory and experiment have provided information about these orbitals, which occupy a place in molecules analogous to the energy region around the Fermi surface in solids at $0 \mathrm{~K}$. Many times the nature of these orbitals is inferred from theory, but experimental approaches are possible. Absorption spectroscopy provides a method for investigating the occupied orbitals of a molecule, with the technique of photoelectron spectroscopy (PES) frequently yielding the most easily interpreted results. ${ }^{2}$

The unoccupied orbitals of a molecule can, in some cases, lead to stable negative ions if an electron is added to one of them. These can be studied by photodetachment, a spectroscopy analogous to PES for neutrals. ${ }^{3}$ In other cases the unoccupied orbitals can lead to temporary negative ions. ${ }^{4,5}$ The most direct method of investigating these cases involves the measurement of the low energy electron scattering from molecules. ${ }^{6-8}$ The presence of the unoccupied orbitals is revealed by structures of varying sharpness in the cross sections. These are called resonances. One can describe the phenomenon by saying that an electron impinging on the molecule during the scattering experiment gets trapped in an unoccupied orbital for a time and then leaves. The time of entrapment is frequently $\sim 10^{-14} \mathrm{~s}$. Although short, this time is still long compared to the orbiting times of outer electrons in molecules $\left(10^{-16} \mathrm{~s}\right)$ and, for this reason, the molecules with the added electron are frequently called temporary negative ions.

When unoccupied orbitals lead to resonances, the term shape resonance is usually used; it comes from recognition that the phenomenon arises because of the shape of the potential barrier that the incoming electron sees. The electron is delayed in the unoccupied orbital because it must tunnel through an angular momentum barrier, both to get in and to get out. The general view is that no resonance structure is seen for unoccupied orbitals that require no tunneling to get into or out of.

From a computational viewpoint, quantum mechanical

\footnotetext{
a) Present address: Department of Chemistry, University of Pittsburgh, Pittsburgh, PA.
}

scattering calculations are considerably more difficult than variational treatments of bound states. In particular, a number of computational packages are available today that provide nearly routine determinations of the self-consistentfield (SCF) energies of atoms or molecules. For systems that are not too large, these calculations approach Hartree-Fock quality.

For molecules, such treatments nearly always use the basis set method. As is well known, there results, when any but an absolute minimal basis set is used, a set of orbitals outside the occupied ones that are termed the virtual orbitals (VO). These have no direct physical significance in the neutral molecule and, indeed, the orbital energies associated with them have no absolute variational stability. An exception to the latter situation will arise if the system treated possesses a stable negative ion with such a large electron affinity (EA) that is predicted to exist at the SCF level of approximation. In some cases, VO energies may have local minima (see Sec. VIII).

Another well-known fact concerning the VOs is that they "see" no Fermi hole in the molecule. This means that they each represent an extra electron that is not really in the neutral molecule. Thus, the VO is associated in some way with a negative ion of the molecule. Koopmans' theorem ${ }^{9,10}$ then gives an approximation to the energy of the system with an added electron. Burrow and Jordan ${ }^{7.8}$ have used this idea with SCF VO energies to help correlate and assign results from resonance measurements by electron transmission experiments. The energies obtained this way are usually too high, but subtracting a constant from the theoretical values to bring one of them into agreement with an experimental result has provided useful comparisons with experiment.

Calculations of the scattering of electrons from $n$-electron molecular systems have been made by a number of methods. Hazi and Taylor ${ }^{11,12}$ pioneered the stabilization method, while recent applications of the method have been made by Nestman and Payerimhoff, ${ }^{13}$ Chao, et al.,${ }^{14}$ and Falcetta and Jordan. ${ }^{15}$ The application of a number of other methods to molecular problems has been reviewed recently by Collins and Schneider. ${ }^{16}$ In principle, any method for dealing with the scattering problem will allow one to discuss the theory of shape resonances in molecules, but we suggest that a particularly useful approach involves the interaction of a discrete state with the continuum. This idea is most often associated with Feshbach ${ }^{17}$ and Fano, ${ }^{18}$ and the latter has 
called the implementation "configuration interaction between a quasibound state and the continuum"' (QBSCCI). One is led to thinking of this method by the nature of the VOs discussed above, and we shall investigate their use in constructing the quasibound state functions of the QBSCCI approach.

\section{OUTLINE OF THEORY}

Using projection operators to separate the Hamiltonian into diagonal and nondiagonal portions, the method of Feshbach $^{17}$ and Fano ${ }^{18}$ gives

$$
\begin{aligned}
& H=H^{0}+V, \\
& H^{0}=|b\rangle E_{b}\left\langle b\left|+\int d E d^{2} \hat{k}\right| E \hat{k}\right\rangle E\langle E \hat{k}|, \\
& V=\int d E d^{2} \hat{k}\left\{|b\rangle V_{b E \hat{k}}\langle E \hat{k}|+| E \hat{k}\rangle V_{E \hat{k} b}\langle b|\right\}, \\
& \langle b \mid b\rangle=1, \\
& \langle b \mid E \hat{k}\rangle=0, \\
& \left\langle E^{\prime} \hat{k}{ }^{\prime} \mid E \hat{k}\right\rangle=\delta\left(E-E^{\prime}\right) \delta\left(\hat{k}-\hat{k}^{\prime}\right), \\
& I=|b\rangle\left\langle b\left|+\int d E d^{2} \hat{k}\right| E \hat{k}\right\rangle\langle E \hat{k}|,
\end{aligned}
$$

where $|b\rangle$ is the wave function corresponding to the quasibound state and $|E \hat{k}\rangle$ is the wave function for the continuum part of the Hilbert space.

As is well known, the Hamiltonian system represented by Eqs. (1)-(7) is exactly soluble. ${ }^{17,18}$ This means that the Lippmann-Schwinger equation for the transition operator

$$
T=V+V\left(E+i 0-H^{0}\right)^{-1} T,
$$

may also be solved exactly, or what is equivalent, the inverse of $\left[1-V\left(E+i 0-H^{0}\right)^{-1}\right]$ may be written exactly in the basis of the resolution of the identity given in Eq. (7). Therefore,

$$
\left\langle E \hat{k}^{\prime}|T| E \hat{k}\right\rangle=\frac{V_{E \hat{k}{ }^{\prime} b} V_{b E \hat{k}}}{E-E_{b}-\Delta(E)+i[\Gamma(E) / 2]},
$$

where the important quantities are the width function

$$
\Gamma(E)=2 \pi \int d^{2} \hat{k}\left|V_{b E \hat{k}}\right|^{2}
$$

and the level shift function

$$
\Delta(E)=\frac{P}{2 \pi} \int d E^{\prime} \frac{\Gamma\left(E^{\prime}\right)}{E-E^{\prime}} .
$$

It will be observed that $\Gamma(E)>0$ from its definition. The sign of $\Delta(E)$, on the other hand, is more complicated to judge. We call attention to the fact that the normalization of the continuum functions in Eq. (6) imply a threshold behavior,

$$
\Gamma(E)=\text { const } \times E^{a} ; \quad a \geqslant \frac{1}{2}
$$

therefore, the principle value integral in Eq. (11) converges as the energy goes to 0 and, necessarily,

$$
\Delta(0)<0 \text {. }
$$

From continuity, we therefore expect a finite energy region
$0 \leqslant E<E_{0}$ for which the level shift is negative. For higher energies, the level shift may stay positive or alternate in sign periodically, but will go to zero as $E \rightarrow \infty$.

Our derivation of the transition matrix ignores the background term arising from $H^{0}$. When this is included, the total transition matrix in terms of the background and the portion from Eq. (9) is

$$
T_{t}=T_{b}+T-2 \pi T_{b} T .
$$

In our applications of Eqs. (9) and (14), we will, however, expect the background term to be small, since the leading $l>0$ and the energy is low. Under these circumstances and for our purposes, we will ignore the background terms in Eq. (14).

\section{THE ROLE OF THE WIDTH AND SHIFT FUNCTIONS}

Ignoring the background contribution, the expression in Eq. (9) has the general appearance of the familiar BreitWigner resonance profile. Nevertheless, the dependence of $\Gamma(E)$ and $\Delta(E)$ on energy can modify the expected cross section significantly. In the discussion that follows in this section, we assume that $E_{b}$ is positive. In this connection, we note that with the energy scale (discussed below) adopted in this article, a negative value for this energy would imply the existence of a variationally stable negative ion of our system. We return below to the calculation of $E_{b}$.

The first question that must be answered is does the equation

$$
E^{\prime}-E_{b}-\Delta\left(E^{\prime}\right)=0
$$

possess a root $E^{\prime}$ that is real and positive? [Here, again, we note that a real negative root of Eq. (15) implies the existence of a stable negative ion.] A real and positive root is by no means guaranteed by the theory. We consider three cases.

\section{A. A sharp resonance}

If $\Gamma(E)$ and $\Delta(E)$ are small, then we expect $E^{\prime}=E_{b}^{\prime} \approx E_{b}+\Delta\left(E_{b}\right)<E_{b}$. If they are also weak functions of the energy, we may approximate Eq. (9) as

$$
\left\langle E \hat{k}^{\prime}|T| E \hat{k}\right\rangle=\frac{V_{E_{b}^{\prime} \hat{k}^{\prime} b} V_{b E_{b}^{\prime} \hat{k}}}{E-E_{b}^{\prime}+i\left[\Gamma\left(E_{b}^{\prime}\right) / 2\right]},
$$

which has the conventional Breit-Wigner form. When $E=E_{b}^{\prime}$, the value of the transition matrix element is pure imaginary, corresponding to a phase $\operatorname{shift}(\bmod \pi)$ of $\pi / 2$ and a maximum in the cross section (ignoring the influence of the background). The actual full width at half-height (FWHH) of the resonance is very nearly $\Gamma\left(E_{b}^{\prime}\right)$. In many polyatomic molecules, vibrational structure will be visible on shape resonances that come under this category if the FWHH is smaller than the nominal vibrational spacing. This can complicate the experimental problem of determining the electronic contribution to $\Gamma\left(E_{b}^{\prime}\right)$, which is all we are concerned with in this article.

\section{B. A broad strong feature}

If the shift function in Eq. (15) is large enough in magnitude and changes rapidly enough, there may be no root of 
the proper sort, or $E-E_{b}-F(E)$ may barely reach zero or barely pass it. In these circumstances, the transition matrix approaches a nearly pure imaginary value and may remain near this for some considerable range of the energy. The corresponding cross section will rise to the vicinity of its allowed maximum and also remain. Because of the $1 / E$ factor in the cross section, the allowed maximum nevertheless falls as the energy increases further; the general appearance is a broad peak in the cross section that may be difficult to see experimentally. The actual origin of this profile is somewhat different from case $\mathbf{A}$ where the resonance falls significantly from its allowed maximum after the center of the peak is passed.

We expect little or no visible vibrational structure on the "resonance" peak in this case. Although any rise in the phase shift implies some delay time for the electron in a state described by $|b\rangle$, it is probably shorter than a vibrational period. ${ }^{19}$ The apparent width of the measured peak is not closely connected to $\Gamma(E)$ in this case, however. Rather, we expect it to be approximately equal to the onset position of the peak. If the onset is at $E_{0}$, the peak height will be approximately $\propto E_{0}$ and the cross section will have fallen to $1 / 2$ this value at the energy $2 E_{0}$. The full width at half-height is therefore approximately $E_{0}$.

\section{No strong feature}

It is possible for $E_{b}$ and $\Delta(E)$ to cooperate so that the transition matrix always has a large real part. Under these circumstances, we expect the cross section to have no sharp distinguishing features. Physically, there is no reason to invoke the state $|b\rangle$. The OBSCCI method has, in this case, merely produced an artificial division of the continuum space into two pieces.

\section{THE "BOUND" STATE}

When a system has only temporary negative ion states, there is, unfortunately, no mathematically unambiguous way to define the state function $|b\rangle$ and physical arguments must be used. It is well known that wave functions for shape resonances have considerable amplitude close to the nuclei and at some distance from the molecule. A region in between, where the effect of the angular momentum barrier is largest, has low amplitude. Therefore, one wants $a|b\rangle$ that is not too diffuse. The argument goes as follows: The overall wave function will be a sum of $|b\rangle$ and an integral over $|E \hat{k}\rangle$. The ideal situation is to arrange that the "inner" part of the wave function is taken care of by $|b\rangle$ and the outer part by the integral over the continuum function. Unfortunately, the energy $E_{b}$ alone is insufficient as a guide to having a good $|b\rangle$. This is easily seen, since, in fact, a normalizable function orthogonal to $|b\rangle$ (which we presume to be a good representation of the inner part of the resonance) can always be written as

$$
|\Phi\rangle=\int d E d^{2} \hat{k} \Phi(E \hat{k})|E \hat{k}\rangle
$$

and, if $\Phi(E \hat{k})$ is sharply peaked in energy around the value $E_{b}$, the expectation value of the Hamiltonian for the function defined in Eq. (17) can also have this same value. In spite of the appropriate energy, because $\langle\Phi \mid b\rangle=0,|\Phi\rangle$ would not be expected to a good approximation for the inner part of the resonant wave function.

\section{v. b) AND THE VIRTUAL ORBITALS OF SCF THEORY}

Linear combinations of Gaussian functions are routinely used today as the atomic orbital (AO) ingredients for linear-combinations-of-atomic-orbital-molecular orbital (LCAO-MO) calculations of the electronic structure of polyatomic molecules. Although ions are sometimes of interest, the overwhelming number of these calculations are for neutral molecules and, usually, the AO bases are optimized for this sort of system. As we noted above, SCF treatments using discrete bases will always produce a set of VOs, unless the basis is truly minimal. Since the VOs are linear combinations of the same AOs that are of an appropriate diffuseness for the neutral valence state orbitals of the molecule, one expects that at least some of the VOs are suitable to construct a function $|b\rangle$ of the sort we need to calculate the electronic properties of a shape resonance.

We now specify that the neutral molecule to be treated is a closed shell system with $n$ electrons in a singlet state. Thus, if we take the point of view in the derivation of Koopmans' theorem, a function $|b\rangle$ appropriate for a shape resonance of the molecule will have one open shell orbital and doublet multiplicity. A single Slater determinant approximation for this is

$$
|b\rangle=N\left|u_{1} \bar{u}_{1} \cdots u_{n} \bar{u}_{n} v_{i}\right|,
$$

where the spin orbitals $x$ or $\bar{x}$ have $m_{s}$ of $1 / 2$ or $-1 / 2$, respectively. Koopmans' theorem tells us that the energy for this function is

$$
E_{b}=E_{\mathrm{SCF}}+\epsilon_{i},
$$

where $\epsilon_{i}$ is the energy of the VO. The energy in Eq. (18) conforms to the convention in $a b$ initio calculations where total energies are used almost universally. This, however, is inconvenient in scattering calculations, where it is more important to focus on the energy of the initially free electron. Thus, we take as our zero the energy of the system when the projectile electron is at infinity and at rest. We subtract $E_{\mathrm{SCF}}$ from Eq. (19), and $E_{b}$ is then just $\epsilon_{i}$. An added advantage is the simplification of the energy limits in integrals in many previous equations. They become 0 to $\infty$. We emphasize that our discussion in Sec. III of the sign of $E_{b}$ and the stability of the negative ion assumes this latter convention for the energy zero.

Of considerable practical importance is the problem of choosing which $V O$ to represent $|b\rangle$. If a large flexible AO basis is used, there will be many VOs and not all will have the characteristics needed. This is particularly true if the AO basis has a number of diffuse orbitals. Because the VOs have no variational stability, some may have $\epsilon_{i}$ that are already lower than the ultimate $E_{b}^{\prime}$ expected for the system. This is no problem, in principle. As long as Eqs. (1), (2), and (3) represent the exact (nonrelativistic) Hamiltonian of the system, the results should be independent of the precise form of $|b\rangle$. Nevertheless, practical exact calculations are rare and we expect choices of physically suitable $|b\rangle$ 's to do better in 
approximate treatments. We give, below, the results of a test of these ideas.

One could choose among the VOs in a number of ways. An examination of the function can show if the amplitude is large in the inner part of the molecule. DeRose, et al., have suggested that expectation values of $\left\langle r^{2}\right\rangle$ can be used to sort the VOs into suitable and unsuitable classes. ${ }^{20} \mathrm{~A}$ variant of this would be to diagonalize the $r^{2}$ operator in the subspace of the VOs and use the eigenfunction of the minimum eigenvalue.

Another way would be to leave extremely diffuse AOs out of the basis for the calculation of the neutral molecule. One would then choose the VO of appropriate symmetry type that has the lowest $\epsilon_{i}$. We use this method for the most part in our calculations described below.

\section{THE $|E \hat{k}\rangle$ FUNCTION}

The continuum function to effect the separation of the Hamiltonian shown in Eqs. (1), (2), and (3) is more difficult to determine. We use the following procedure:

A single determinantal function with one electron occupying a plane wave function provides the primitive basis for the continuum function

$$
\left|\epsilon^{\prime}\right\rangle=\sqrt{\frac{k}{8 \pi^{3}}} N\left|u_{1} \bar{u}_{1} \cdots u_{n} \bar{u}_{n} \exp (i \bar{k} \cdot \bar{r})\right|,
$$

where we have specified an energy delta-function normalization. The orthogonality [Eq. (5)] of the continuum functions with the bound function requires projecting out $|b\rangle$ :

$$
|\epsilon\rangle=\left|\epsilon^{\prime}\right\rangle-|b\rangle\left\langle b \mid \epsilon^{\prime}\right\rangle \text {. }
$$

$|\epsilon\rangle$, in turn, must be modified further. The actual function for out purposes may be written in terms of the off-the-energy-shell K matrix

$$
\begin{aligned}
|E \hat{k}\rangle= & {\left[|\epsilon\rangle+\frac{1}{\pi} \int d E^{\prime} d^{2} \hat{k}^{\prime}\right.} \\
& \left.\times \frac{\left|\epsilon\left(E^{\prime} \hat{k}^{\prime}\right)\right\rangle K\left(E^{\prime} \hat{k}^{\prime}, E \hat{k}\right)}{E-E^{\prime}}\right] \cos \delta_{b},
\end{aligned}
$$

where the $\mathbf{K}$ matrix is a solution of the integral equation

$$
\begin{aligned}
K\left(E^{\prime} \hat{k}^{\prime}, E \hat{k}\right) & \\
= & -\pi V\left(E^{\prime} \hat{k}^{\prime}, E \hat{k}\right) \\
& -\int d E^{\prime \prime} d^{2} \hat{k}^{\prime \prime} S\left(E^{\prime} \hat{k}^{\prime}, E^{\prime \prime} \hat{k}^{\prime \prime}\right) K\left(E^{\prime \prime} \hat{k}^{\prime \prime}, E \hat{k}\right) \\
& -\int d E^{\prime \prime} d^{2} \hat{k}^{\prime \prime} \frac{V\left(E^{\prime} \hat{k}^{\prime}, E^{\prime \prime} \hat{k}^{\prime \prime}\right) K\left(E^{\prime \prime} \hat{k} ", E \hat{k}\right)}{E^{\prime \prime}-E},
\end{aligned}
$$

$S$ and $V$ are defined by

$$
\begin{aligned}
\left\langle\epsilon\left(E^{\prime} \hat{k}^{\prime}\right) \mid \epsilon(E \hat{k})\right\rangle= & \delta\left(E^{\prime}-E\right) \delta\left(\hat{k}^{\prime}-\hat{k}\right) \\
& +S\left(E^{\prime} \hat{k}^{\prime}, E \hat{k}\right)
\end{aligned}
$$

and

$$
V\left(E^{\prime} \hat{k}^{\prime}, E \hat{k}\right)=\left\langle\epsilon\left(E^{\prime} \hat{k}^{\prime}\right)|H-E| \epsilon(E \hat{k})\right\rangle,
$$

and $\delta_{b}$ is the background phase shift. We must, of course, use the exact (nonrelativistic) Hamiltonian in Eq. (25). It is shown elsewhere ${ }^{21}$ that the continuum function defined by this procedure along with the "bound" state function actually satisfies the closure equation (7).

It might legitimately be asked why we introduced at all the notion of the bound state $|b\rangle$ when, in principle, Eqs. (22) $-(25)$ by themselves provide a solution to our scattering problem if they are applied directly to $\left|\epsilon^{\prime}\right\rangle$ of Eq. (20 rather than to Eq. (21). The answer contains two parts: (1) The solution of Eqs. (22)-(25) for cases where $\mathbf{K}$ is rapidly changing can be quite difficult. This certainly includes situations where there are shape resonances. In contrast, Eq. (9) was easily obtained. (2) The introduction of the bound state function is clearly warranted by the physics of the situation. It separates the expression for the scattering amplitude into a resonant part and a background in a way that, in ideal cases, allows one to ignore the background. Indeed, in the latter parts of this article, we shall assume that $\delta_{b}$ in Eq. (22) is small enough to be approximated by zero, or what is equivalent, $\mathbf{K}$ in Eq. (22) is assumed negligible in the appropriate energy regions.

Once the virtual orbital used to construct $|b\rangle$ has been chosen, the argument above allows one to approximate the crucial quantity of the theory

$$
\begin{aligned}
V_{b E \hat{k}}= & {\left[\langle b|H| \epsilon(E \hat{k})\rangle+\frac{1}{\pi} \int d E^{\prime} d^{2} \hat{k}^{\prime}\right.} \\
& \left.\times \frac{\left\langle b|H| \epsilon\left(E^{\prime} \hat{k}^{\prime}\right)\right\rangle K\left(E^{\prime} \hat{k}^{\prime}, E \hat{k}\right)}{E-E^{\prime}}\right] \cos \delta_{b} \\
\approx & \langle b|H| \epsilon(E \hat{k})\rangle .
\end{aligned}
$$

In the Appendix we outline our procedure for evaluating the last integral in Eq. (26).

\section{APPLICATION TO THE $\mathrm{H}_{2}$ MOLECULE}

We give an application of the above theory to the $\mathbf{H}_{2}$ molecule, examining the ${ }^{2} \Sigma_{u}^{+}$temporary negative ion. We calculate the SCF function for the molecule using a triplezeta polarization (ZP) basis set by Dunning and Hay. ${ }^{22}$ With this basis, we obtain $4 \sigma_{q}$ and $4 \sigma_{u}$ orbitals and contour plots of the $\sigma_{u}$ orbitals we might choose to construct $|b\rangle$ are shown in Fig. 1. We expect the orbital $1 \sigma_{u}$ to best satisfy the requirements for the quasibound state function. Nevertheless, we have calculated the phase shifts for all of them to illustrate the sensitivity to the particular orbital chosen. The $2 \sigma_{u}$ orbital has a radial node, the $3 \sigma_{u}$ two such nodes, and the $4 \sigma_{u}$ orbital has an unusual shape, somewhat reminiscent of an $f_{\sigma}$ AO. Table I gives some details of the orbital energies for this calculation.

We show in Fig. 2 the low energy phase shifts generated when the present theory is used with each of the four $\sigma_{u}$ VOs from this calculation. As is seen, the first three, which have manifestly $\sigma_{u}$ character, all give phase shifts quite close to one another. This is in spite of the large differences between the $E_{b}$ values for the functions. $4 \sigma_{u}$, on the other hand, shows a strikingly different result. We thus have the satisfying situation that the phase shifts are reasonably insensitive to the precise form of the $|b\rangle$, as long as the VO in it is not too outlandish. The remaining discrepancies in the first three cases may be due to the approximation in $\mathrm{Eq}$. (26) as well as 

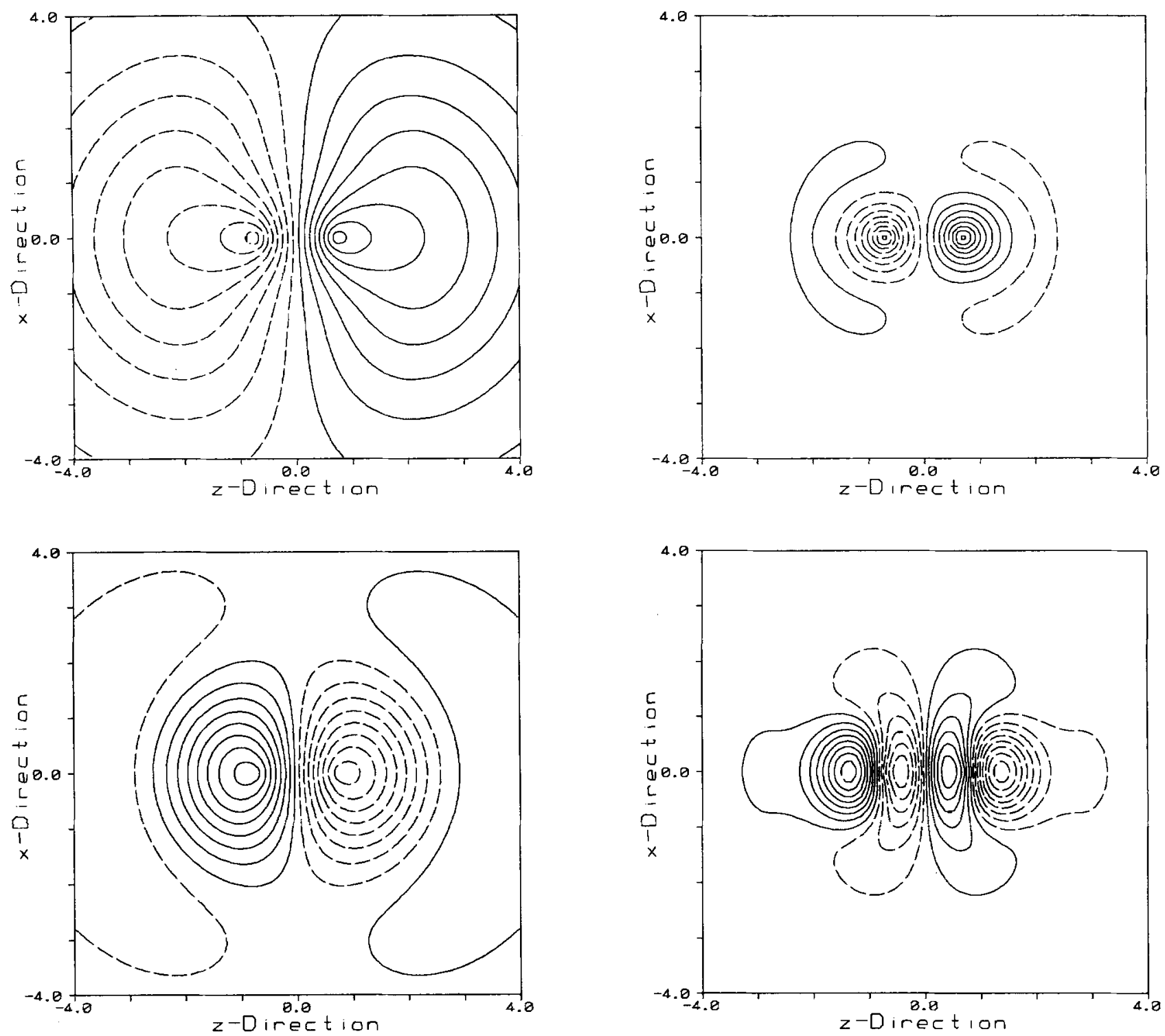

FIG. 1. Contour drawings of the four $\sigma_{u}$ VOS of $\mathrm{H}_{2}$ : (a) $1 \sigma_{u}$; (b) $2 \sigma_{u}$; (c) $3 \sigma_{u}$; and (d) $4 \sigma_{u}$. In each case, there are 16 contours from the maximum to the minimum value. The solid and dashed lines represent the positive and negative contours, respectively. Also in each case, the contours are from values on a plane containing the two nuclei. The edges of the graphs are marked in bohr.

truncation errors in the numerical evaluation of the integral in Eq. (11).

Examination of Fig. 2 shows that the phase shift in this case corresponds to case B of those discussed in Sec. IV. According to these calculations, the phase shift never quite reaches $\pi / 2$ and no sharp peak should be seen in the cross section. In the neighborhood of the rise in the phase shift, the value of $d \delta / d E$ implies a delay time $\sim 75$ atomic time units $\left(2 \times 10^{-15} \mathrm{~s}\right)$. Comparing this with the vibrational period $\sim 10^{-14} \mathrm{~s}$, one expects no vibrational structure in elastic scattering from $\mathrm{H}_{2}$.

\section{THREE HYDROCARBONS $\mathrm{C}_{2} \mathrm{H}_{4}, \mathrm{C}_{3} \mathrm{H}_{4}$, AND $\mathrm{C}_{4} \mathrm{H}_{6}$}

We have calculated the shape resonances of ethylene, cyclopropene, and cyclobutene using essentially the same method. The experimental position and width for ethylene is given by Burrow and Jordan, ${ }^{7}$ and the values for the ring compounds have been determined by Howard and Staley. ${ }^{23}$ The principal feature we have added to the calculation of these hydrocarbons involves a sort of stabilization treatment of the VO that is involved in the resonance. We describe the procedure specifically for ethylene.

We have argued above that too diffuse a basis set will yield VOs that do not probe the correct part of the molecular space. With this in mind, we consider the treatment of these hydrocarbons with the fairly restricted basis of STO-3G AOs. ${ }^{24}$ We augment this basis with a single component Gaussian $p$ function at each of the carbon atoms. The scale factor of this orbital has relatively little influence on the SCF energy of the neutral molecule, but the effect on the VO is significant. We show in Fig. 3 the variation of the energy of 
TABLE I. The orbital energies of the $\sigma_{u}$ virtual orbitals for the SCF calculation of $\mathbf{H}_{2}$.

\begin{tabular}{cr}
\hline \hline Orbital & Energy (eV) \\
\hline $1 \sigma_{u}$ & 5.238 \\
$2 \sigma_{u}$ & 20.996 \\
$3 \sigma_{u}$ & 88.977 \\
$4 \sigma_{u}$ & 121.788 \\
$E_{\text {SCF }}$ & -30.834 \\
\hline
\end{tabular}

the $1 b_{2 g}$ VO of ethylene with the exponential scale factor of the added single Gaussian function. It is seen that although there is clearly no variational stability, a local minimum in the energy appears at a scale factor of $0.268\left(\mathrm{bohr}^{-2}\right)$. This behavior is similar to the "stabilization" phenomenon in the total energy of a system proposed by Haze and Taylor as a method to determine resonances. ${ }^{1}$ In this case, we do not use the energy curve of Fig. 3 directly to get resonance parameters, but use the VO, at the local minimum, to construct the function $|b\rangle$ for our treatment. There is a similar local minimum in the corresponding curves for cyclopropene and cyclobutene at the scale factors 0.284 and 0.276 , respectively.

Table II shows our results for the positions and widths of the low-lying shape resonances of the three unsaturated hydrocarbons we are discussing. These are all associated most closely with the antibonding $\pi$ orbital in the neighborhood of the $\mathrm{C}=\mathrm{C}$ double bond. Comparisons with other calculations and experiment are also given. Considering the approximations in the present treatment, the agreement with ethylene and cyclopropene is good, while cyclobutene shows a greater discrepancy. Nevertheless, in all cases, the effect of the energy shift brings the parameters from the scattering calculation closer to experiment than does the simple application of Koopmans' theorem.

The difficulties with cyclobutene are not clear and identifying their origin will require more work. The too small width and the too small energy shift go together, indicating that $V_{b E \hat{k}}$ is too small. Further observations are probably premature.

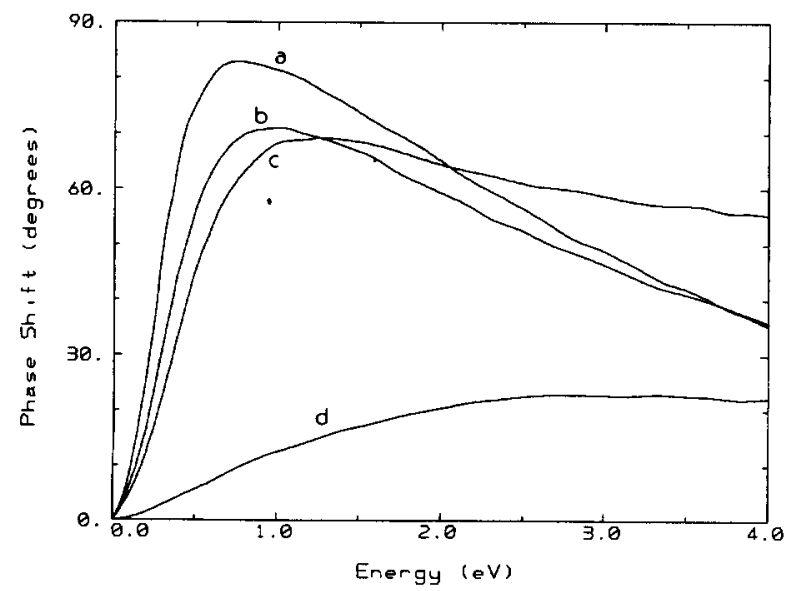

FIG. 2. The calculated phase shifts for the ${ }^{2} \Sigma_{g}^{+}$resonance of $H_{2}$. The letters marking the curves correspond to those in the caption to Fig. 1.

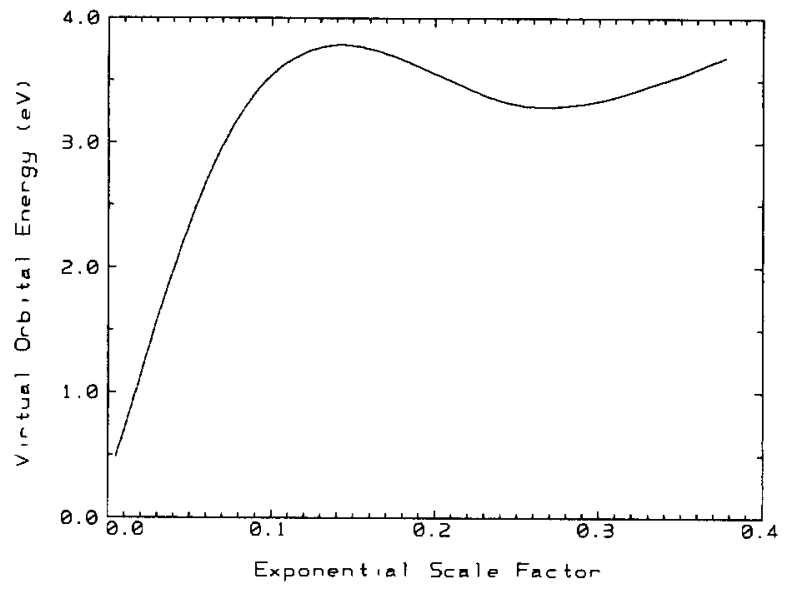

FIG. 3. The "stabilization" curve of the $p-\pi$ STO-3G orbitals in the basis for the ethylene calculation.

Howard and Staley also examined more closely the exact relation between the VOenergies and the resonance positions. ${ }^{23}$ For a series of cyclic hydrocarbons, using the 6-31G basis, they discovered a reasonably good linear relationship between these two types of quantities. Heinrich, et al..$^{25}$ have also considered this sort of correlation between calculated and measured quantities. We make a further analysis of such correlations in Sec. $\mathrm{X}$.

\section{THE $\mathbf{N}_{\mathbf{2}}$ MOLECULE}

We finish with a discussion of the $\mathrm{N}_{2}$ molecule. This molecule has been the subject of more studies, both theoretical and experimental, than any other. Much of the interest has centered around the detailed nature of the vibrational structure. Early studies of the electronic part of the resonance were by Gilmore, ${ }^{26}$ and Krauss and Mies. ${ }^{27}$ For recent developments, see Burke and West. ${ }^{28}$

The treatments of molecules discussed heretofore in this article have all involved a single determinant $|b\rangle$ function. For $\mathrm{N}_{2}$, we compare the single determinant calculation with one where we represent the bound state with a multiconfiguration function consisting of the reference function and single excitations from the $\pi_{u}$ valence orbitals into appropriate VOs. Table III shows our results at different levels of theory compared with other calculations and empirical

TABLE II. Comparison of experimental and calculated quantities associated with shape resonances in three unsaturated hydrocarbons.

\begin{tabular}{lcccccc}
\hline \hline & & & \multicolumn{3}{c}{ This calculation } \\
\cline { 4 - 7 } Molecule & $\begin{array}{c}\text { Exp. (Position) } \\
(\mathrm{eV})\end{array}$ & $\begin{array}{c}6-31 \mathrm{G}^{\mathrm{a}, \mathrm{b}} \\
(\mathrm{eV})\end{array}$ & $\begin{array}{c}\mathrm{SCF}^{\mathrm{a}, \mathrm{c}} \\
(\mathrm{eV})\end{array}$ & $\begin{array}{c}\text { Position } \\
(\mathrm{eV})\end{array}$ & $\begin{array}{c}\text { Width } \\
(\mathrm{eV})\end{array}$ \\
\hline Ethylene & $1.74^{\mathrm{d}}$ & 4.72 & 3.30 & 1.83 & 0.69 \\
Cyclopropene & $1.73^{\mathrm{b}}$ & 4.71 & 3.12 & 1.81 & 0.66 \\
Cyclobutene & $2.00^{\mathrm{b}}$ & 4.94 & 3.31 & 2.73 & 0.28 \\
\hline \hline
\end{tabular}

'Koopmans' theorem.

${ }^{\mathrm{b}}$ Reference 23.

'Stabilized value (see the text, Sec. VIII).

d Reference 7. 
parameters obtained from experiment. ${ }^{29}$ The calculations were carried out at the experimental equilibrium nuclear separation using a Dunning double-zeta polarization (DZP) basis. ${ }^{22}$

The single configuration result is comparable to the static exchange calculation of Schneider ${ }^{30}$ The principal consequences of including excited configurations in $\mathrm{N}_{2}$ are a partial relaxation of the core and a lowering of the value of $E_{b}$. This results in placing $E_{b}^{\prime}$ at a value where $\Gamma\left(E_{b}^{\prime}\right)$ is smaller than that resulting from the single configuration calculation. Such a treatment includes effects similar to the relaxed core $R$-matrix calculation of Schneider, et al. ${ }^{31}$ This clearly improves the comparison with experiment. The added physics of electron-molecule interaction being described in the multiconfiguration function involves mainly spin polarization. ${ }^{32,33}$ Because of the Pauli principle, the repulsion between the electrons of the molecule and the projectile electron with the same $m_{s}$ value is different from the repulsion in the case where the signs are opposite. The single configuration function corresponding to Koopmans' theorem, with its perfect pairing of double occupied orbitals, cannot describe this phenomenon. A multiconfiguration function is required and single excitations provide the most important contribution to this effect. We also include in Table III results from somewhat different multiconfiguration stabilization calculations by Nestman and Peyerimhoff, ${ }^{13}$ and by Chao, et al. ${ }^{14}$ We discuss these results further in Sec. XI.

\section{CORRELATIONS BETWEEN VO ENERGIES AND RESONANCE POSITIONS}

Correlations between VO energies using various basis sets and experimental values of resonance positions have been made by Howard and Staley, ${ }^{23}$ and Heinrich, et al. ${ }^{25}$ As noted above, the correlation diagram shows a reasonably close clustering about a straight line. Taking these data for the 6-31G basis, a conventional least-squares fit of a straight line gives

$$
E_{\mathrm{vo}}=1.31 E_{\text {exp }}+2.33(\mathrm{eV}),
$$

where $E_{\exp }$ is the experimental energy and $E_{\mathrm{Vo}}$ is the VO orbital energy. Comparing Eq. (27) with Eq. (15), we can

TABLE III. The ${ }^{2} \Pi_{g}$ shape resonance in $\mathrm{N}_{2}$ from various calculations and experiment.

\begin{tabular}{lccc}
\hline \hline $\begin{array}{l}\text { Bound state } \\
\text { function }\end{array}$ & $\begin{array}{c}E_{b} \\
(\mathrm{eV})\end{array}$ & $\begin{array}{c}E_{\mathrm{rec}} \\
(\mathrm{eV})\end{array}$ & $\begin{array}{c}\Gamma \\
(\mathrm{eV})\end{array}$ \\
\hline Single configuration $^{\mathrm{a}}$ & 5.026 & 3.8 & 1.5 \\
Multiconfiguration $^{\mathrm{a}}$ & 2.924 & 1.8 & 0.39 \\
Static exchange $^{\mathrm{b}}$ & $\cdots$ & 3.70 & 1.16 \\
$R$ matrix $^{\mathrm{c}}$ & $\cdots$ & 2.15 & 0.34 \\
Stabilization $^{\mathrm{d}}$ & $\cdots$ & 2.62 & 0.45 \\
Stabilization $^{\mathrm{e}}$ & $\cdots$ & 2.44 & 0.32 \\
Empirical $^{\mathrm{e}}$ & $\cdots$ & 2.20 & 0.54 \\
\hline \hline
\end{tabular}

a The present calculation.

${ }^{\mathrm{b}}$ Reference 30.

${ }^{\mathrm{c}}$ Reference 31 .

${ }^{\mathrm{d}}$ Reference 13.

'Reference 14.

${ }^{\mathrm{r}}$ Reference 29. identify the experimental energy with $E$ and the VO energy with $E_{b}$. Thus, we obtain

$$
\Delta(E)=-2.33-0.31 E
$$

as a crude estimate of the shift function.

A fitting of the VO vs experimental data to a more sophisticated expression for the shift function is useful, however, and we set up a model function for $\Gamma(E)$. From the expression for $V_{b E \hat{k}}$ given in the Appendix, we expect $\Gamma(E)$ to be zero at $E=0$, to rise to a maximum, and then generally fall to zero as some power $E^{-n}$. Since $V_{b E \hat{k}}$ is expected to oscillate, there will be places where $\Gamma(E)$ also is zero, but these will not seriously affect our qualitative argument. Domcke and Cederbaum, ${ }^{34}$ and Berman, et al.${ }^{35}$ have used exponential expressions to model width functions and we continue this practice with the simple expression

$$
\Gamma(E)=\Gamma_{\max }\left[\frac{E}{E_{\max }} \exp \left(1-\frac{E}{E_{\max }}\right)\right]^{l+1 / 2},
$$

where $\Gamma_{\max }$ is the maximum value taken on by the function and $E_{\max }$ is the energy where the maximum occurs. Using Eqs. (11) and (29), we obtain an expression for the shift function

$$
\Delta(E)=-\frac{\Gamma_{\max }}{2 \pi} e^{(l+1 / 2)} W_{l}\left(\frac{E}{E_{\max }}\right),
$$

where

$$
\begin{aligned}
W_{l}(x)= & \frac{x^{l}}{\sqrt{l+1 / 2}}\left\{\sum_{k=0}^{\prime-1} \frac{\Gamma(k+3 / 2)}{[(l+1 / 2) x]^{k+1}}\right. \\
& +\sqrt{\pi}-\pi \Re[i \sqrt{x} w(i \sqrt{x})]\},
\end{aligned}
$$

and $w(z)=e^{-z^{\prime}} \operatorname{erfc}(-i z)$ is the "complex" error function. ${ }^{36}$

Varying $E_{\max }$ and $\Gamma_{\max }$ and using a least-squares criterion, we have obtained a best fit of the function $E-\Delta(E)$ from Eq. (30) for $l=1$ to the data of Heinrich, et al..$^{25}$ The best values are $E_{\max }=15.6 \mathrm{eV}$ and $\Gamma_{\max }=7.0 \mathrm{eV}$. A comparison of the calculated function and the data is shown in Fig. 4 , where it is seen that there is good qualitative agreement.

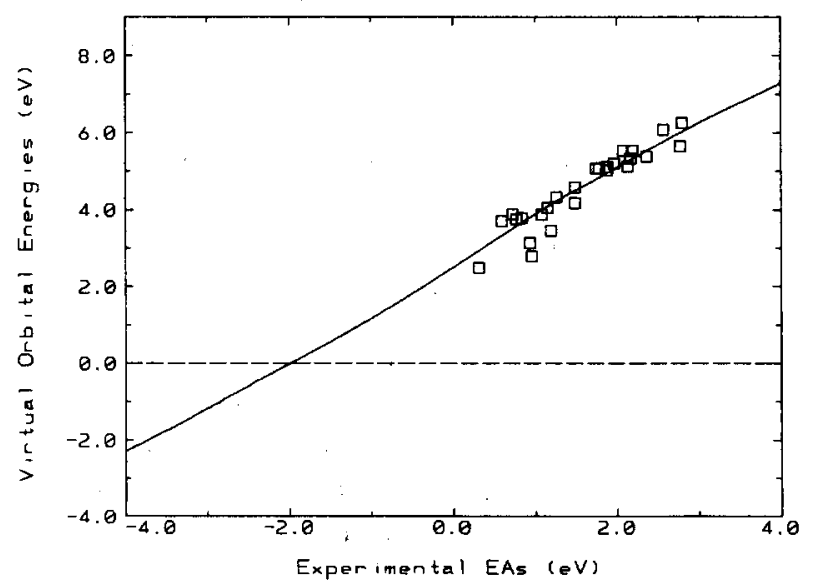

FIG. 4. A comparison of the calculated function $E-\Delta(E)$ from Eq. (29) and the data of Heinrich, et al. (Ref. 25). The function is evaluated for the best values $E_{\max }=15.6 \mathrm{eV}, \Gamma_{\max }=7.0 \mathrm{eV}$, and an arbitrarily selected value of $l=1$. 
The SCF calculation provides no estimate of resonance widths; we therefore cannot make any sort of corresponding correlation between calculated and experimental widths.

We emphasize that the result of the fitting shown in Fig. 4 is specific to the $6-31 \mathrm{G}$ basis set and possibly to the present set of molecules. Nevertheless, this procedure may provide a method for converting VO energies for a wider range of substances. Further studies will be required to test whatever range of validity is provided by an expression such as Eq. (30).

\section{DISCUSSION}

Koopmans' theorem occupies a central place in the treatment of this article. Proof of the theorem is based upon the unrestricted Hartree-Fock (UHF) procedure. ${ }^{10}$ The restricted Hartree-Fock (RHF) procedure, as discussed by Roothaan, ${ }^{37}$ is frequently used in discussing molecules, however. The one sort of state where the UHF and RHF procedures give the same result is, of course, the closed shell singlet, which is the only one we have treated. For open shell systems, the RHF procedure, although it has, unlike the UHF, the great advantage of yielding pure spin states, does not give virtual orbital energies in the same sense as does the UHF and does not have a Koopmans-like theorem. Thus Koopmans' theorem applies to the closed shell RHF wave function because, for this case only, the orbitals represent a stationary point of the UHF energy functional, as well as of the RHF functional.

In the closed shell case, discrepancies between experiment and the predictions of Koopmans' theorem are attributed to relaxation and correlation energy effects that are ignored in the theory. As is well known, these are of a sign so that some cancellation occurs between these errors when a molecule is ionized by the loss of an electron. On the other hand, these two effects will tend to augment one another when a temporary (or stable) negative ion is formed. We thus expect Koopmans' theorem to yield values for $E_{b}$ that are too high in energy by the sum of the errors mentioned and, consequently, the calculated position of the resonance should also be too high. Nevertheless, the calculated values for the resonance positions of the smaller hydrocarbons in Table II are in good agreement. We note, also, that the calculated resonance position for $\mathbf{N}_{2}$ in the multiconfiguration treatment is lower than experiment.

These results suggest that the energy shift $\Delta(E)$ we calculate is too large in magnitude. We suggest that a significant contribution to this error is the approximation made in $\mathrm{Eq}$. (25), where the background $K\left(E^{\prime} \hat{k}^{\prime}, E \hat{k}\right)$ matrix is ignored. Preliminary calculations of scattering in $\mathrm{H}_{2}$ show that the magnitude of $V_{b E \hat{k}}$ is reduced in the lower energy regions when $K\left(E^{\prime} \hat{k}^{\prime}, E \hat{k}\right)$ is not ignored ${ }^{38}$ If this is universally true, $\Gamma(E)$ would in every case also be reduced in magnitude. Consequently, we expect the widths to be somewhat smaller and the energy shifts less extreme. Clearly, further work will be required to substantiate this idea.

When we consider the empirical correlations of VO energies with measured resonance positions, the deficiencies in Koopmans' theorem cannot be ignored. Thinking of the energy shift function as a correction to convert the calculated energies to experimental ones, our fitted $\Delta(E)$ corrects for the relaxation and correlation errors as well as for the spatially restricted nature of the $|b\rangle$ function. Of course, only the latter is its proper role. There seems little we can do about this in the absence of a general theory for molecules relating spin and charge polarization to molecular structure. It is perhaps surprising, nonetheless, that the correlation between calculated and measured energies is as linear as it is. This possibly indicates that the errors in Koopmans' theorem are nearly proportional to the predicted energies. Again, these ideas require more work to confirm or disprove.

\section{SUMMARY}

Using the quasibound state continuum configuration interaction (CI) theory of Feshbach ${ }^{17}$ and Fano, ${ }^{18}$ we have considered the relationship between the virtual orbitals of $a b$ initio SCF theory and resonances due to temporary negative ions of diatomic and polyatomic molecules. Specifically, $\mathrm{H}_{2}$, $\mathrm{N}_{2}$, ethylene, cyclopropene, and cyclobutene have been treated. A number of important conclusions can be drawn:

(a) The virtual orbitals of SCF theory provide reasonable one electron functions to represent the extra electron in $n+1$ electron functions for the quasibound state. Within limits and as long as the basis does not contain extremely diffuse functions, the results are insensitive to the precise choice of virtual orbital.

(b) The energy shift function, typical of this sort of resonance theory, it qualitatively of the right size and sign to bring the calculated virtual orbital energies in line with measured positions of resonances. It provides both a shift and a compression in the scale.

(c) Among the molecules studied here, the simple single configuration theory is most inadequate for $\mathrm{N}_{2}$. In this case, a first order $\mathrm{CI}$ representation of the quasibound state function improves the agreement between theory and experiment. The principal effect of the first order CI is the inclusion of spin polarization.

(d) An empirical function relating calculated virtual orbital energies and experimental resonance positions provides an empirical energy shift function that has a potential for universality.

\section{APPENDIX}

In this Appendix, we show the calculation of the matrix element $V_{b E \hat{k}}$. From Eqs. (18), (20), and (21), we have $V_{b E \hat{k}}=\langle b|H| \epsilon(E \hat{k})\rangle=\left\langle b|H| \epsilon^{\prime}(E \hat{k})\right\rangle$

$$
-\langle b|H| b\rangle\left\langle b \mid \epsilon^{\prime}(E \hat{k})\right\rangle \text {. }
$$

Remembering that the VO in the $|b\rangle$ is orthogonal to the other occupied orbitals, the Slater-Condon rules for determining the matrix elements of determinantal functions give

$$
V_{b E \hat{k}}=\langle v|F| c(\bar{k})\rangle-E_{b}\langle v \mid c(\bar{k})\rangle,
$$

where $c(\bar{k})=\sqrt{k / 8 \pi^{3}} \exp (i \bar{k} \cdot \bar{r})$ is the plane wave continuum "orbital", $v$ is the VO used to construct the quasibound state function, and $F$ is the standard Fock operator for the neutral molecule, which in this case is assumed to be in a closed shell singlet state. ${ }^{37}$ In deriving Eq. (A2), we have used the relation 


$$
\left\langle v|F| u_{i}\right\rangle=0 ; i=1, \ldots, n,
$$

which is true because the Fock operator is diagonal in the basis consisting of the occupied and virtual orbitals of the neutral system.

The calculation of $V_{b E \hat{k}}$ for the single excitation function in the case of $\mathrm{N}_{2}$ requires the evaluation of more general matrix elements than that of Eqs. (A1), but similar techniques apply.

The above expressions give the integral in a $\mathbf{k}$ representation. Where needed, we have used numerical techniques to expand the quantity in spherical harmonics to obtain the integral in an $l m$ representation.

'H. Fujimoto and K. Fukui, Isr. J. Chem. 19, 317 (1980).

${ }^{2}$ See, e.g., Principles of Ultraviolet Photoelectron Spectroscopy, edited by $\mathbf{J}$. W. Rabalais (Wiley, New York, 1977).

${ }^{3}$ See, e.g., H. Hotop, T. A. Patterson, and C. W. Lineberger, Phys. Rev. A 8, 762 (1973); J. Simons and K. D. Jordan, Chem. Rev. 87, 535 (1987).

${ }^{4} J$. N. Bardsley, A. Herzenberg, and F. Mandl, Proc. R. Soc. London Ser. A 89, 321 (1966).

${ }^{5}$ N. F. Lane, Rev. Mod. Phys. 52, 29 (1980).

'G. J. Schulz, Rev. Mod. Phys. 45, 423 (1973).

${ }^{7}$ K. D. Jordan and P. D. Burrow, Acc. Chem. Res. 11, 341 (1978).

${ }^{8}$ K. D. Jordan and P. D. Burrow, Chem. Rev. 87, 557 (1987).

${ }^{\text {T}} \mathrm{T}$. Koopmans, Physica 1, 105 (1933).

${ }^{10}$ S. T. Epstein, The Variation Method in Quantum Chemistry (Academic, New York, 1974), p. 45.

"A. U. Hazi and H. S. Taylor, Phys. Rev. A 1, 1109 (1970).

${ }^{12}$ H. S. Taylor and A. U. Hazi, Phys. Rev. A 14, 2071 (1976)

${ }^{13}$ B. Nestman and S. D. Peyerimhoff, J. Phys. B 18, 615 (1985): 18, 4309 (1985).
${ }^{14}$ J. S. -Y. Chao, M. Falcetta, and K. D. Jordan, J. Chem. Phys. 93, 1125 (1990).

${ }^{15}$ M. Falcetta and K. D. Jordan, J. Phys. Chem. 94, 5666 (1990).

${ }^{16} \mathrm{~L}$. A. Collins and B. I. Schneider, in Electron-Molecule Scattering and Photoionization, edited by P. G. Burke and J. B. West (Plenum, New York, 1988) and references therein.

${ }^{17}$ H. Feshbach, Ann. Phys. NY 5, 357 (1958).

${ }^{18}$ U. Fano, Phys. Rev. 124, 1866 (1961).

${ }^{19}$ Scattering Theory, edited by J. R. Taylor (Wiley, New York, 1972), Sec. 13-c.

${ }^{20}$ E. DeRose, E. A. Gilason, and N. H. Sabelli, J. Chem. Phys. 82, 4577 (1985).

${ }^{21}$ G. A. Gallup, J. Phys. B 23, 2383S, 2397S (1990).

${ }^{22}$ T. H. Dunning and J. F. Hay, in Methods of Electronic Scattering Theory, edited by H. F. Schaefer (Plenum, New York, 1977) pp. 1.

${ }^{23}$ A. E. Howard and S. W. Staley, in Resonances, edited by D. G. Truhlar (American Chemical Society, Washington, D.C. 1984).

${ }^{24}$ W. J. Hehre, R. F. Stewart, and J. A. Pople, J. Chem. Phys. 51, 2657 (1969).

${ }^{25}$ N. Heinrich, W. Koch, and G. Frenking, Chem. Phys. Lett. 124, 20 (1986).

${ }^{26}$ F. R. Gilmore, J. Quantum Spectrosc. Radiat. Transfer 5, 369 (1965).

${ }^{27}$ M. Krauss and F. H. Mies, Phys. Rev. A 1, 1592 (1970).

${ }^{28}$ Several articles in Electron-Molecule Scattering and Photoionization, edited by P. G. Burke and J. B. West (Plenum, New York, 1987).

${ }^{29}$ L. Dubé and A. Herzenberg, Phys. Rev. A 20, 194 (1979).

${ }^{30}$ B. L. Schneider, Phys. Rev. A 24, 1 (1981).

${ }^{31}$ B. L. Schneider, M. Le Dourneuf, and V. K. Lan, Phys. Rev. Lett. 43, 1927 (1979).

${ }^{32}$ H. Kollmar and V. Staemmler, Theor. Chim. Acta 48, 223 (1978).

${ }^{33}$ G. A. Gallup, J. Chem. Phys. 86, 4018 (1987).

${ }^{34}$ W. Domcke and L. S. Cederbaum, Phys. Rev. A 16, 1465 (1977).

${ }^{35}$ M. Berman, C. Mundel, and W. Domcke, Phys. Rev. A 31, 641 (1985).

${ }^{36} \mathrm{M}$. Abramowitz and I. Stegun, Handbook of Mathematical Functions (Dover, New York, 1965), Def. 7.1.3.

${ }^{37}$ C. C. J. Roothaan, Rev. Mod. Phys. 23, 69 (1951).

${ }^{38}$ Unpublished results. 